\title{
Mentoring Doctoral Students within a Virtual Community of Practice - With Special Reference to Their Information Recording Behaviour
}

\author{
Pieter A. Van Brakel \\ Cape Peninsula University of Technology, \\ Cape Town, South Africa
}

vanbrakelp@cput.ac.za

\begin{abstract}
Master's and doctoral students in particular are expected to demonstrate advanced research skills with regard to identifying, evaluating, recording and using information for research, especially their skills in analysing already published information which might be utilised to document the status quo of a particular aspect of a topic (sometimes called the literature study).. There are various ways and means to guide or coach advanced post-graduate students at masters and doctoral level. Once such approach is to work from within a virtually community of practice (VCoP). Because of the nature of learning content, additional benefits can be created. This paper will argue and demonstrate why a $\mathrm{VCoP}$ is the ideal environment to mentor doctoral students in certain aspects of their research (for example identifying a research problem, selecting investigative research questions, or writing a literature review). A theoretical framework as departure point will illustrate how knowledge transfer (or coaching) effectively takes place within a VCoP. The paper will then focus on information recording techniques as a case study. The latter is one of the most neglected areas in the information seeking process. The author will also demonstrate how mindmapping software can provide in an effective way to record or store information segments by means of multi-tier keywords. Examples of mind mapping for information recording will also be provided.
\end{abstract}

Keywords: Virtual communities of practice, VCoPs, information sharing, knowledge sharing, information recording, post-graduate research.

\section{Introduction}

When the intricacies the research process and its dynamics are researched the inexperienced researcher will soon discover that during this process multiple activities has to be managed properly. The intrinsic nature of these phases has been described by a multitude of sources on research

Material published as part of this publication, either on-line or in print, is copyrighted by the Informing Science Institute. Permission to make digital or paper copy of part or all of these works for personal or classroom use is granted without fee provided that the copies are not made or distributed for profit or commercial advantage AND that copies 1) bear this notice in full and 2) give the full citation on the first page. It is permissible to abstract these works so long as credit is given. To copy in all other cases or to republish or to post on a server or to redistribute to lists requires specific permission and payment of a fee. Contact Publisher@InformingScience.org to request redistribution permission. methodologies. A basic countdown of these phases may look as follows (the order may differ, depending on the experience of the reader):

- Doing a basic literature search for recently-published information within a discipline in view of a process called 'topic discovery' 
- Formulation of a research problem and selecting research objectives and investigative questions

- Setting up a research plan, indicating a research methodology (research design)

- Writing a research proposal

- Executing a comprehensive literature search

- 'Reading' relevant literature (plus 'note-taking' of relevant sections of the literature)

- Writing a 'critical review' of the literature

- Embarking the empirical section of the research

- Reporting on the empirical findings using qualitative or quantitative research methods

- Write an interpretation and conclusion.

Needless to state, the theory of actually implementing these phases is probably being taught from even the undergraduate stages of learning, and in a more advanced mode during postgraduate programmes. It can also be stated without fear of contradiction that every supervisor, even those with limited supervisory expertise will know the importance of creating a research design which will ensure that the research process and its phases is correctly planned and executed. Furthermore, various teaching methods and techniques are being utilized in teaching the skills to execute these phases, from formal lectures to seminars to workshops to group discussions, or to the individual during a supervisor's personal discussions with a candidate.

This paper is based on extensive experience of the author in supervising postgraduate students (the term $P G$ researcher is preferred and will be used throughout this paper). A major point to mention here is that irrespective of various formal or short courses in research design and research methodology, and irrespective of research seminars and workshops, the supervisor must still continuously and repeatedly be on standby in dispensing ideas, facts, hints and even (again!) resources to consult on research. This 'consultation process' invariably has to occur during personal and regular visits by individuals, which is exceptionally unproductive and time-consuming, to say the least. From the PG researcher's point of view, note taking must (again) takes place, plus further reading if necessary.

The premise of this paper is that the theory and practice of a sub-section of the so-called literature review is often neglected in sources about research methods, even in sources that specifically describe the management of research-based information (see for example the latest edition of Orna and Stevens: 2009). These and other general works on research methods (examples will be given below) do well in discussing all the other processes that are related to the literature review (literature analysis, for example), but the techniques described by the authors to note or document what has been read and classified as relevant for later use, is mostly non-existent. It is for this reason that this topic (information recording) will be brought in below to illustrate what role the VCoP can play in the PG teaching and learning process.

At first, the pros and cons of establishing a virtual community of practice (VCoP) was investigated. A major motivation for embarking into the possibilities of electronic communication is that the current generation of users of online systems is increasingly more proficient in social networking. Soon after implementation the efficiency of PG researchers has become a major support system in this author's supervision activities, and specifically (as mentioned above) in developing a PG researcher's information recording skills. A VCoP consisting of PG researchers is the ideal environment to coach them with regard to any aspect in the research process. The premise of this paper is therefore that VCoPs provide in an informal but effective sphere of information and knowledge sharing: between supervisor and candidates, and between candidates themselves. 
The following research questions will therefore be addressed in this paper:

- Why is a VCoP ideal for coaching PG researchers?

- What are the requirements for efficient and time-effective information recording?

- How can these requirements be addressed with mind map software?

\section{VCoPS as Enablers of Knowledge Sharing}

\section{Defining and Positioning VCoPS}

It is common knowledge that the phrase community of practice can be attributed to Lave and Wenger (1991, p. 98) who described it as an activity '... about which participants share understandings concerning what they are doing and what that means in their lives and for their community'. This fits well into the PG research domain, namely that they share certain theories with which the group members can execute mutual tasks. A long list of authors can be cited that have built upon Lave and Wenger's. It is not the purpose of this paper to extensively report on these, but rather to selectively identify certain features only, thus providing reasons why a VCoP can work well in the PG research domain. In this context Koh and Kim's interpretation is also worthwhile, namely that a community (of practice) can be seen as a group where individuals come together based on an obligation to one another or to 'gather' for a shared purpose (2004, p. 156). Furthermore, the nature of virtual communities is the fact that the less experienced members of the community can learn from the contributions of the more mature and experienced members. Newcomers eventually perform minor 'tasks' or activities in the completion of a major assignment, but eventually they will avail themselves for more important contributions or parts thereof (Ardichvili et al., 2006, p. 95).

It follows that knowledge and specifically knowledge sharing can be identified here as being an essential element within a community which is being supervised or rather coached for PG research. In general VCoP terms, of the three crucial sectors of a community network (knowledge, people, and social network) knowledge is described as the key factor in sustaining a VCoP (Fang and Chiu, 2010, p. 236). In fact, knowledge-sharing has long been regarded as motivation for using virtual communities. Ardichvilli et al (2006, p. 95) go one step further by stating that knowledge is the key element in sustaining organizational competitiveness.

Apart from the argumentation that knowledge is what is exchange and shared, it is also important to take note of the type of knowledge featuring within a VCoP. We typically distinguish between explicit and tacit knowledge. The first category refers to knowledge that is easy to communicate, that has been articulated in written format and stored within a 'system' that can be easily transmitted to others. Tacit knowledge is difficult to communicate as it is invariably linked to the experience of the provider. It is mostly intuitive and embedded in a specific context. It is difficult for others to imitate or copy tacit knowledge. Ardichvili et al. (2006, p. 95) quote Wenger (2002) and colleagues with regard the problematic sharing of tacit knowledge. In itself, tacit knowledge requires active interaction amongst individuals, especially in the form of storytelling. It can therefore be argued that VCoPs can here act as a platform for storytelling exchanges to take place. In PG research 'storytelling' takes place when one individual shares particular positive or even negative experience in their research, for example when selecting a sample or creating a research design.

Lastly, this researcher has found yet another concept that can be brought into the theory of this domain: communities of learning. As long ago as 2001 Johnson quoted several authors to conclude that VCoPs trace their roots to constructivism (2001, p. 47). In this work he listed various 
elements that can move control of learning from instructors to learners, for example by adapting the traditional lecturer:student model with actions such as

- ill-structured problems (for example in open-ended questions)

- learning in social and physical context of real-world problems

- shared goals

- cognitive tools

- instructor's role as facilitator or coach (the word mentor wil also work here).

It is probably the VCoP of PG researchers which can most aptly demonstrate the importance of a constructivistic approach of teaching and learning. The examples in the case study below will demonstrate this statement.

In a study by Gannon-Leary and Fontainha (2007) multiple authors were cited to illustrate how a VCoP can be beneficial to sharing and learning. It shows clearly that learning within these communities are collaborative, in which the collaborative knowledge of the community is greater than any individual knowledge. Furthermore, Lave and Wenger (1991) are of the opinion that learners (or PG researchers for that matter) participating in a VCoP are assimilated into the socio-cultural practices of the community to gain knowledge and skills from those community members who act as masters. A constructivistic approach should also be related to the critical success factors listed below: most of not all of those CSFs rely on the skills and experience of the supervisor as coach. The example below of how a topic such as information recording can be enfolded within the $\mathrm{VCoP}$ will illustrate best how cognitive tools can be employed to assist learners (researchers) to share and organize knowledge.

\section{Barriers to Effective Knowledge Sharing}

Certain barriers to the effective exploitation of a virtual community have been identified and should be kept in mind in the knowledge sharing process (Gannon-Leary \& Fontainha, 2007); only those relevant to this paper are listed and interpreted as follows:

- Subject knowledge: Learning content might be too advanced or could even be classified as cutting edge knowledge. If this is the case it may be possible that only a select thus very small group of members of the community can take part. It will also imply that less learning takes place as some building blocks are missing from the novices in the group. In a PG research group it is therefore important to expose new topics from the basics to a more advanced level.

- Academic freedom and collegiality: Motivation to join a VCoP may be weak where a high collegiality exists, namely a strong community of people who are physically working together. 'Face-to-face communication is easier, why should one be 'forced' to exchange ideas via an online community' the question may be asked. A strong and cohesive PG group is not confined to the same physical environment - they are invariably working from different environments, even from different areas or countries.

- Fluid in its composition: Membership shifting refers to the fact that a high level of energy is maintained so that the members thereof stays stimulated and eager to share their knowledge on topics that are brought into the realm of discussions. High relevant topics should continuously feed into the community; examples are being discussed below with regard to information recording, eg the role of information recording in life-long learning.

- Trust: Trust building is vital for knowledge sharing, and develops traditionally through faceto-face interaction. In a virtual environment a supervisor should apply mechanisms to not only create trust within the PG research group, but also maintain a high level of confidence in the 
honesty and potential quality of the participants. For example, the PG group can be introduced to each other (brief biographical outline) and kept in the system for future reference.

- Misinterpretation of messages: Today's ICTs are not always flawless with regard to stability and therefore lack the richness of face-to-face communication. The geographical gap between participants can lead to misinterpretation as non-verbal cues may be lost or hidden (for example file attachments not delivered, different software programmes and/or different versions within the community). In the example describe below, special care should be taken to ensure that the PG group is using the same mindmap software program, otherwise individual contributions might be limited to a few.

\section{Critical Success Factors}

To conclude the theoretical section of this paper, one could state that most if not all of the abovementioned barriers are removed the chances for success in using a VCoP for knowledge sharing within a learning environment are sealed. If this can be achieved the following critical success factors will enhance a supervisor's contribution to the development of his or her PG research group (Gannon-Leary \& Fontainha, 2007):

- Technology and usability: Participants should have access to and essential IT skills to become efficient virtual community members. Today this is a basic requirement as provided by the typical tertiary institute.

- Communication: Fundamental in the development of trust, the latter allows the VCoP to grow, change and achieve its objective. A VCoP for PG research is of such a nature that most if not all participants have a common purpose, might know each other or most members, and can easily build up trust with a supervisor.

- Membership: Prior knowledge of each other, and/or an awareness why each other belong to the group assist in consolidating membership and developing trust. In a PG research group the members could each develop a personal blog, or have a Face Book entry with personal biographical information.

- Sense of belonging: This can be challenging to overcome in a cross-national and cross-cultural online community. Consideration needs to be given how different cultures may perceive a certain instruction or assignment. In an interactive group this could certainly develop but the supervisor should be aware of the implications of cultural differences as in correlation to expecting assignments for knowlegde sharing.

- Sense of purpose: A virtual CoP must have a sense of purpose and this purpose must be achievable via the ICTs being utilised. PG research supervision incorporates a relative closed group, all with the same purpose, namely to successfully complete their theses in the minimum of time.

- User-friendly language: Unexplained jargon being used in e-communication can put off the uninitiated if definitions or a terminology list does not exist within a VCoP. In research terms quite a useful list can be developed with time and saved in a link available to the PG group.

- Time breeds longevity: Time is needed both for optimal communication and to build up trust and a sense of belonging. A virtual community of postgraduate students displays ample motivation and dedication towards a mutual purpose. Furthermore, if a doctorate student stays with the same supervisor, he or she stays within the VCoP for even a longer time period. Longevity can even be further strengthened if a completed doctorate is appointed as post-doc in a department, or as research assistant for the supervisor. 
All of the above CSFs can be relatively easy accomplished in an enclosed environment such as explained in this paper, namely research students situated around a single supervisor, all with the purpose to complete their research as effective as possible.. However, it does not mean that the moderator of the $\mathrm{VCoP}$ (supervisor) should not proceed with caution as trust in the quality of knowledge being shared within a VCoP is of the utmost importance. The next section describes a case study as identified in the research problem for this paper, namely how effective techniques of recording information for the literature analysis of a research project could be presented.

\section{Case Study: Information Recording Techniques}

\section{The Literature on Information Recording}

Scanning the literature on research methodology, it is quite remarkable that not much deliberation has gone into the theory and practice of a system of systematically recording the relevant sections of a particular information source.

Information recording is a term used by this author for this action (also referred to terms such as summarising, note taking, keyword indexing). Notetaking is often used, although in later work the concept of management of research information is more often used. This might have been after Orna and Stevens' book, of which a second re-worked edition appeared recently (2009). For this paper two prominent (and popular) books have been chosen to illustrate the apparent lack of thought going into this very essential phase of research; both addresses a distinct approach namely the inexperienced researcher, and both written by experienced supervisors:

- Bell: Doing your research project: a guide for first-time researchers in education, health and social science) - obviously written for the younger researcher, this work calls on the researcher to '...make notes of what seem to you to be important issues and highlight them' (2006:62). Further on (pp 72-73) reference is made to the complexity of finding '... ways in which you will be able to find who wrote what about different topics.' Tragically no further solutions are being offered, except than referring to but not discussing a 'card index' or computer program to organise references.

- Punch: Developing effective research proposals - quite an extensive work, addressing the preparation and creation of a research proposal from topic discovery to examples of good proposals. Interesting enough, although a number of pages are devoted to 'relevant literature' (pp. 42-45) in the whole book nothing is discussed on how relevant and usable sections from the literature could be recorded.

\section{A New Approach}

This brings us back to Orna and Stevens' work referred to in the previous paragraph (2009), which is currently the most extensive with regard to the comprehensive process of managing research-base information. In their Chapter 3 (Information to support research - and how to manage it) the topic 'Making it findable: keys to the information store' is brought to the fore. (p. 6981 ). In a nutshell this very valuable section discusses the option to record all source retrieved, then add keywords that describe (index) the content of the source (pp. 73-74). If this approach negate the use of 'summaries' or 'note taking' it is not mentioned anywhere by the authors, except to broadly add the content of a particular information source on the bibliographic record (cards or a bibliographic database).

It is imperative that PG researchers apply the most effective techniques in recording useable sections from relevant documents. It is therefore essential to expose them to a technique or set of techniques that can meet the following criteria: 
- No time-consuming summarisation of potentially relevant information

- Systematic investigating and exploring of 'unknown territory'

- Effective (systematic) recording of new content elements

- Direct link between reference and content element

- Easy tracking of content gaps

- 'Birds-eye view' of progress in reading for the literature review

- Logic enfolding or development of theoretical chapters

- Finding of small sections or elements of content at the writing phase.

This author has developed the idea of keyword assigning one step further as none of the current techniques described above can match the criteria mentioned in the bulleted list. The approach can be described as a multi-tier keyword approach and functions as follows:

- Use mind map software or $2 \times 3$ cards to plot the system.

- Assign a specific (narrow) keyword to each relevant content element (one sentence; a few paragraphs; a section; some pages), that is potentially relevant in solving the research problem.

- Add 2-4 broader (wider) keywords to create a contextual or systematic set of keyword levels.

- On the record (3x5 card; mind map; any other software) add only a abbreviated reference, for example the author, date of publication and page number on the entity (record) of the most specific keyword ); that is, the keyword on the far-right.

The following example briefly illustrates the headings moving from broad (far left) to very narrow, thus the concept of a typical multi-tiered approach:

Example: Breeding tendencies of birds of prey

Vultures / Breeding / Incubation / Cape vulture

Vultures / Breeding / Incubation / Palmnut vulture

Vultures / Breeding / Incubation / Egyptian vulture

Vultures / Breeding / Nest sites / Palmnut vulture

Vultures / Material / Nest sites / Cape vulture

Eagles / Breeding / Incubation / Martial eagle

Eagles / Breeding / Nest sites / Martial eagle

Instead of cards mind map software is an effective replacement of the card system. Apart from being more versatile, it contains multiple additional functionalities such as

- export possibilities to other MS software

- use of colour

- possibility of adding hyperlinks and symbols to internal and external sources referred to in relation to the most specific keyword

- much clearer exposition of keyword levels

- easier to change (typical need as more knowledgeable.

Figure 1 replicates the example given above ('birds of prey'). Gradually, as the literature is searched and read, digital cards enfolds, with references being added to the very last card in the line. Once the reading process has been completed, both for the purposes of a research proposal and later the comprehensive literature analysis, the map should not only exhibit the complete structure (and references) of the literature being read, but also exposing the gaps in the structure of the thesis or research paper. The researcher's literature analysis and writing up can now start. 


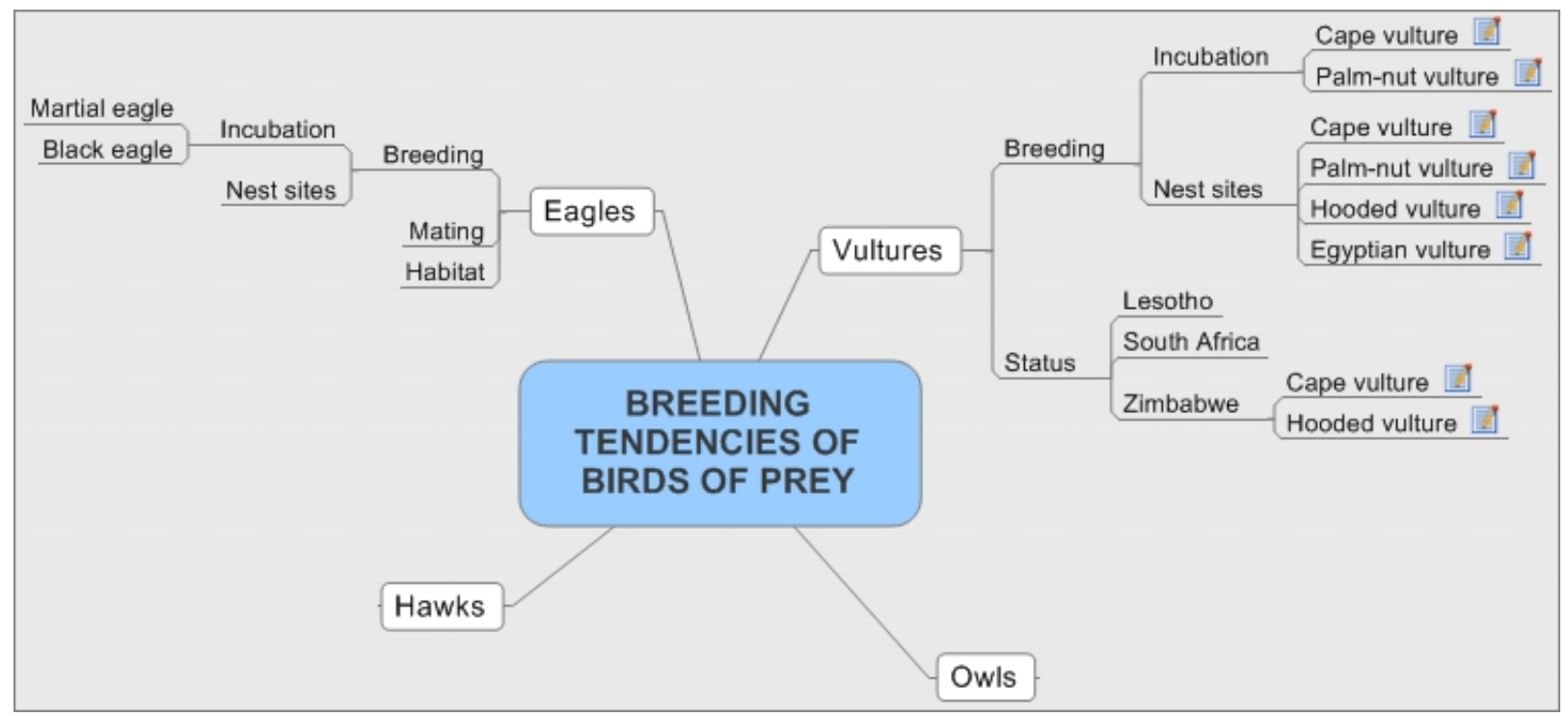

Figure 1: Mind-mapping with a muli-tier keyword approach

\section{Coaching Information Recording in a VCoP}

In a virtual circle of active PG researchers different levels of competencies always exist with regard to the research approach. Also, different levels of progress always exist (from first year postgraduate enrolments to those who are working towards the closing sections of their theses). Although at first this could be seen as a problem, in a VCoP this is actually a huge benefit. When defining virtual networks earlier in this paper Lave and Wenger (1991) was quoted as saying that '...learners (or PG researchers for that matter) participating in a $\mathrm{VCoP}$ are assimilated into the socio-cultural practices of the community to gain knowledge and skills from those community members who act as masters.' In this case the senior members or 'masters' (experienced doctorals and the supervisor) work in close collaboration to share knowledge.

The following list of communications to the PG researchers illustrates some examples of 'illstructured questions' (assignments) put to this author's virtual community of PG researchers. Knowledge sharing takes place irrespective of the progress or qualification level of the participant:

Welcome message from the supervisor at the beginning of the academic year, indicating items such as:

- Expectations for research within the group

- Funding for the project

- Emphasis of the importance of taking part in the research process

- Invitation to share individual knowledge

- Outline of discussion points, such as

$\checkmark$ reading to establish a research problem

$\checkmark$ formulating a field and title for the research

$\checkmark$ writing a research proposal

$\checkmark$ establishing research design

$\checkmark$ techniques for a comprehensive literature search (e-indexes)

$\checkmark$ and more 
Effective information recording for a research proposal:

- Approach and intensity of searching for literature needed at this level

- Literature searching techniques

Shortcomings of traditional single-keyword approaches

- Disadvantages of summaries

- Principles of the single keyword approach (in tandem with a bibliographic databases in view of referencing)

A multi-tier approach (see above under the heading A New Approach)

- Principles of the approach

- Pros and cons working with a card system

- Pros and cons working with a mind map (or similar) program

PG group's feedback on information recording: Various comments and contributions are being stored - herewith a selection from the responses to illustrate the essence of the knowledge being shared between the VCoP members:

- 'Never do summaries - total waste of time. The question always arise: "How comprehensive must a summary be"? In the end one can just as well keep the complete article or source'.

- Summaries are problematic to store; how do you organise them if more than one subtopic is discussed.

- It is important to decide on or develop an information recording system before any reading - even for a research proposal - starts.

- A mult-tier approach works best if a separate bibliographic database is maintained, containing author details as well as keywords for retrieval via two to five separate keywords; this is to later retrieve the complete document.

- A muti-tier approach creates the opportunity to combine brainstorming with this information recording approach.

- I prefer 3x5 cards for more visibility as well as mobility.

- A program such as for mindmapping is easily changed, relations and positions of branches moved and added.

- Take the results of your information recording with when visiting your supervisor. A mindmap allows for a printout of only the substructure or chapter to be discussed (for example Chapter 3).

- Always keep backups of your information recording, even if working from cards (photocopying is an effective and time-saving solution).

- Remember that any of your presentation or reports can be prepared by using a multi-tier approach. Even in your future work-related responsibilities.

\section{Conclusion}

Master's and doctoral students in particular are expected to demonstrate advanced research skills with regard to identifying, evaluating, recording and using information for research. One skill is invariably neglected, that is their capabilities in analysing already published data and information which might be utilised to document the status quo of a particular aspect of a topic. The literature does not address this problem effectively. In this paper the author has therefore embarked on introducing a new method to records small, medium or large sectors of usable information in a easy-to-use system: 
- Making summaries obsolete (ineffective and time-consuming)

- Copying and pasting links to relevant sections onto disk (effective in savings in paper, effective with regard to accessing specific (narrow) sections; effective in preventing a reseacher from plagiarism).

A multi-tier keyword approach is most effectively coached or mentored via a VCoP of PG researcher. The theory behind this approach as well as implementing it via a mindmap software program was also described and supported with examples.

\section{References}

Ardichvili, A., Maurer, M., Li, W. Wentling, T. \& Stuedemann, R. (2006). Cultural influences on knowledge sharing through online communities of practice. Journal of Knowledge Management, 10(1), 94107.

Bell, J. (2006). Doing your research project: A guide for first-time researchers in education, health and social science (4th ed.). New York: Open University Press.

Fang, Y-H., \& Chiu, C-M. (2010). In justice we trust: Exploring knowledge-sharing continuance intentions in virtual communities of practice. Computers in Human Behaviour, 26, 235-246.

Gannon-Leary, P., \& Fontainha, E. (2007) Communities of practice and virtual learning communities: Benefits, barriers and success factors. eLearning Papers 5. Online at http://www.elearningpapers.eu

Johnson, C. M. (2001). A survey of current research on online communities of practice. The Internet and Higher Education, 4(1), 45-60.

Koh, J., \& Kim, Y-G. (2004). Knowledge sharing in virtual communities: An e-business perspective. Expert Systems with Applications, 26, 155-166.

Lave, J., \& Wenger, E. (1991). Situated learning: Legitimate peripheral participation. Cambridge, USA: Cambridge University Press.

Orna, E., \& Stevens, G. (2009). Managing information for research. Berkshire: Open University Press.

Punch, K. F. (2005). Developing effective research proposals. London: Sage.

Wenger, E., McDermott, R., \& Snyder, W. (2002). Cultivating communities of practice: A guide to managing knowledge. Boston: Harvard Business School.

\section{Biography}

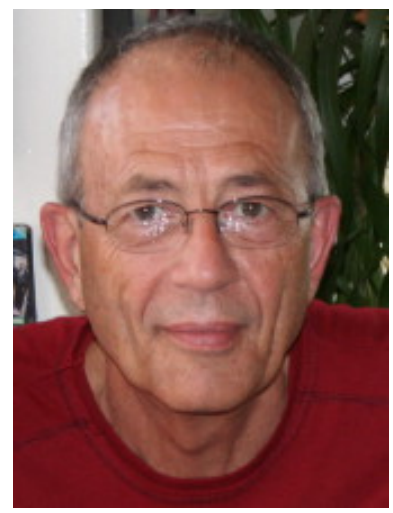

Pieter van Brakel is a professor in the Faculty of Informatics and Design at the Cape Peninsula University of Technology in Cape Town, South Africa. He is currently responsible for this faculty's postgraduate's support office. He is the initiator and the editor of the peerreviewed South African Journal of Information Management since its beginning in June 1999 (see www.sajim.co.za). He initiated and organises since 1999 the Annual Conference on World Wide Applications (see www.za3.co.za), which has been endorsed as a Regional Conference of the International World Wide Web Conference Committee (www.iw3c2.org). Pieter has a masters in Information Science from the University of the Free State, a doctorate in Information Science from the University of Pretoria and a Postgraduate Diploma in Tertiary Education (also from UP). He has published 62 peer-reviewed journal articles and supervised \pm 40 masters and doctoral theses. His research area is virtual communities of practice within private enterprise. 